\title{
Textural Hardness of Selected Ugandan Banana Cultivars under Different Processing Treatments
}

\author{
Samuel Gafuma ${ }^{1}$, G. W. Byarugaba-Bazirake ${ }^{1} \&$ Ediriisa Mugampoza ${ }^{1}$ \\ Dept. of Food Technology, Kyambogo University, P.O Box 1 Kyambogo, Kampala, Uganda \\ Correspondence: G. W. Byarugaba-Bazirake, Dept. of Food Technology, Kyambogo University, P.O Box 1 \\ Kyambogo, Kampala, Uganda. Tel: 256-0776-426578. E-mail: gwbbazirake@ gmail.com
}

Received: June 5, 2018

Accepted: June 22, $2018 \quad$ Online Published: August 14, 2018

doi:10.5539/jfr.v7n5p98

URL: https://doi.org/10.5539/jfr.v7n5p98

\begin{abstract}
Textural hardness affects cooking time, processing, fuel used and the quality of cooked bananas. In this study, textural hardness of selected Ugandan cooking and juice banana cultivars at green maturity was determined using a Texture Analyzer in raw form and at 30, 50, 70, 90, 100 and 130 min in boiled, steamed, mashed and cooled forms.

Raw juice bananas (JB) were significantly harder $(36.17 \mathrm{~N}$ to $42.43 \mathrm{~N})$ than raw cooking bananas (CB) (22.37N to $26.72 \mathrm{~N})(\mathrm{p}<0.05)$. On cooking, JB were harder than CB irrespective of cooking method and time. Boiling and steaming rapidly decreased hardness of the bananas in the first $30 \mathrm{~min}$ and decreased slowly thereafter. Boiling produced softer bananas than steaming while mashing resulted in intermediate hardness. Amongst JB, Kayinja was significantly harder than Ndiizi and Kisubi in boiled and steamed forms ( $<<0.05)$. Hardness of CB was not significantly different $(\mathrm{p}>0.05)$ for all cooking treatments, but Kibuzi was consistently softer while Kazirakwe and Nakabululu were harder than other CB cultivars.

Cooling significantly increased $(\mathrm{p}<0.05)$ hardness of bananas under all treatments with JB being harder in all cases. Mashed and steamed bananas were harder than boiled bananas when cooled. Bananas cooked longer had lower hardness regardless of cooking method.

Overall, textural hardness decreases with cooking time regardless of cooking method. Boiled bananas are softer than mashed or steamed. Cooling increases hardness which follows first order kinetics. Therefore, bananas should either be boiled or steamed and mashed for softer texture and be eaten within $30 \mathrm{~min}$ of serving. Juice bananas should not be cooked because of the hard texture established in this study.
\end{abstract}

Keywords: boiling, cooled bananas, cooking bananas (CB), juice bananas (JB), mashing, steaming, textural hardness

\section{Introduction}

\subsection{Background}

Bananas belong to the family Musaceae (genus Musa) and are a highly perishable crop produced in the tropical and subtropical countries of the world where they are mostly grown for home consumption and local markets. Bananas are subdivided into cooking cultivars (Musa spp AAB and ABB) and non-cooking cultivars (Musa spp AA and AAA) (Perrier et al., 2011). They are consumed as desert fruit; prepared for consumption as food by cooking and roasting; or as a snack by drying; or extracted for juice and fermented for the production of alcoholic beverages such as beer, wine, and gin. Bananas are hence categorized into desert, cooking, juice/beer and roasting bananas depending on their use. According to the Food and Agricultural Organization of the United Nations (FAO), by 2013, banana was the main fruit in international trade and the banana trade figures continue to grow. Based on production quantities, bananas are the most popular fresh fruits worldwide followed by apples and grapes and are second in terms of production volume after watermelons. In 2016, the volume of bananas produced worldwide reached approximately 113.28 million tons down from 115.24 million tons in 2015 (The Portal for Statistics (Statista), 2018). According to FAO statistics, in 2011, about 107 million metric tons of bananas were produced in more than 130 countries representing a total trade value of US\$ 9 billion (Bakare, Ogunbowale, Adegunwa \& Olusanya, 2016). These figures indicate steady growth in banana production worldwide. Banana is the fourth most important food crop in the world after rice, wheat and maize in terms of 
gross domestic product. India is the largest producer of bananas in the world with an annual production of 29 million metric tons (FAO, 2018) from an area of 0.709 million ha.

By 2013, Uganda was the world's largest producer and consumer of cooking bananas with an estimated per capita consumption of 250 - 480kg (Kabahenda \& Kapiriri, 2010). Bananas occupy the largest cultivated area among staple food crops in Uganda and are primarily grown on small subsistence farm plots of average 0.5 ha (Nalunga, Kikulwe, Nowakunda, Ajambo, \& Naziri, 2015). Bananas provide income and are fast becoming a cash crop for Ugandans. Most banana varieties grown in Uganda are endemic to the East African highlands, a region recognized as a secondary center of banana diversity. The East African highland banana is a unique genomic group, selected over centuries by farmers. As many as 84 distinct varieties of endemic East African highland bananas, classified into six clone sets i.e. Nfuuka, Nakyetengu, Nakabululu, Musakala, Ney Poovan $\underline{\mathrm{AB}}$ and Bluggoes $\underline{\mathrm{ABB}}$, are grown by farmers in the region (Karamura 1998). A typical Ugandan household grows averagely as many as 7 banana varieties simultaneously in the banana grove, with a maximum of 27 (Edmeades, Smale, \& Karamura, 2006).

Despite the high production volumes, banana processing remains largely limited to cooking for domestic meals, juice/beer, dried chips and fried crisps. The rest are consumed as dessert. In Uganda, processing of bananas into solid-state shelf-stable products is rare. The reasons for this trend are not well known but textural hardening of cooked bananas could be a major challenge. Globally, texture remains the main sensorial criterion when evaluating the taste and acceptability of bananas (Baoxiu, Moore, \& Orchard, 2000). When cooking bananas are cooked, they soften, but harden immediately on cooling leading to loss of the desirable soft texture and taste. Hardness or softness is a key sensory characteristic for bananas. Softness is important in determining a good cooking plantain cultivar depending on the methods of cooking and use of plantains (Dzomeku, Bam, Adu-kwarteng, Darkey \& Ankomah, 2007a). Hardness affects cooking time and the amount of fuel used. A short cooking time to reach acceptable softness is an advantage where fuel is scarce (Almazan, 1990). Bananas and plantains are cooked in different ways (Dury, Bricas, Tchango-Tchango, \& Bikoi, 2002). In Uganda, bananas are cooked by boiling, steaming combined with mashing or roasting.

Understanding texture characteristics of bananas allows for control of processing operations such as heating (boiling, steaming, mashing, frying, drying etc) and processing time to attain the desired quality attributes of the end product (Chen \& Opara, 2013a). Data on textural hardness of bananas is needed to develop solutions to the textural hardening phenomenon that occurs in cooked bananas and inform consumers about banana varieties that produce tender meals. This information could also be applicable to product development models in case of banana products that are desired globally. However, there is scanty information on textural hardness of Uganda's cooking and juice banana cultivars. Therefore, the objectives of this study were to i) determine the textural hardness of selected Uganda's indigenous cooking and juice bananas when raw, cooked and cooled ii) study the influence of different processing treatments (i.e. boiling, steaming, mashing and cooling) on the textural hardness of bananas ii) examine the influence of cooking time on the textural hardness of cooked bananas.

\section{Materials and Methods}

\subsection{Materials}

Unripe mature green East African Highland Bananas (Musa AAA-EA) endemic to Uganda were used in this study. Eleven cooking banana cultivars selected from four clone sets i.e. Nfuuka clone set (Namande \{NAMD)\}, Nakawere $\{\mathrm{NAKW}\}$ \& Namweezi $\{\mathrm{NAMZ}\}) ;$ Nakitembe clone set (Nakitembe $\{\mathrm{NAKT}\}$, Nakyetengu $\{\mathrm{NAKY}\}$ \& Kibuzi $\{\mathrm{KIB}\}) ;$ Musakala clone set (Musakala \{MUS\}, Mpologoma \{MPO\} \& Kisansa \{KIS\}); and the Nakabululu clone set (Nakabululu $\{\mathrm{NAKB}\}, \&$ Kazirakwe $\{\mathrm{KAZ}\})$ were purchased from Kawanda Agricultural Research Institute (KARI). Three juice banana cultivars were also selected i.e. apple banana or Ndiizi (NDI) and Kisubi (KISB) from the Ney Poovan AB clone set and Kayinja (KAY) from Bluggoes ABB clone set (Karamura, 1998). Ndiizi was bought from KARI while KISB and KAY were obtained from a local farm in Wobulenzi, Luweero District.

\subsection{Sample Harvesting}

Before harvesting, dry matter content and "pulp to peel ratio" of all cooking bananas were monitored for three weeks until when these parameters were constant. All bananas were visibly examined for size and color of the flesh to ensure they had reached maturity. The flesh of all cooking bananas was orange-yellow as determined visually. All banana samples were harvested between 8.00 and 10.00 am and immediately transported to the Department of Food Science \& Nutrition, Makerere University for texture analysis. Analysis of textural hardness was performed within three hours after harvest to avoid influence of respiration on banana texture. 


\subsection{Sampling and Preparation of "Raw Banana Samples"}

For determination of textural hardness of raw bananas, samples were taken by plucking two banana fingers randomly from the bottom, middle and top of the bunch. The fingers were manually washed using potable water and immediately thinly peeled. Peeling was done by making a longitudinal slit along each banana finger and using the knife to carefully open and strip off the skin without removing the surface pulp. Samples were placed on a piece of aluminium foil and transferred to the Texture Analyzer for measurement of hardness.

\subsection{Preparation of "Boiled Banana Samples"}

Boiling of bananas was conducted according to (Paciulli et al., 2016) with modifications. A mixture of banana fingers from the top, middle and bottom of the bunch were randomly picked, washed and peeled. Peeled banana fingers were added to boiling water in a stainless steel pot at a temperature of about $98^{\circ} \mathrm{C}$ and completely covered in a 1:2 bananas to water ratio. The water level was marked and the cooking pot covered with the lid and the bananas boiled on gas flame using an Automatic Ignition Gas system (stainless steel, FL423GC/Gas stove Flamingo, India). Boiling was continued for $30,50,70,90,110$ and $130 \mathrm{~min}$ at $97-98^{\circ} \mathrm{C}$ for all samples. The water level in the cooking pot was maintained by topping up using boiled water prepared at the side. At the end of each time point, two banana samples were picked and placed on a piece of aluminium foil and immediately transferred to the texture analyzer platform for analysis. Three measurements (1 to 1.5 " apart) were performed and recorded for each sample.

\subsection{Preparation of "Steamed Banana Samples"}

A random mixture of banana fingers from the top, middle and bottom of the bunch were picked, washed and peeled. Steamed bananas were prepared by placing the peeled fingers into the middle chamber of a 3 Tier Food Steamer (HS6000, Black \& Decker, China). Samples were steamed for 30, 50, 70, 90,110 and 130 min at 98 $99^{\circ} \mathrm{C}$ and at each sampling time interval two banana fingers were removed, placed on a piece of aluminium foil and transferred to the Texture Analyzer platform for analysis. Three measurements (1 to 1.5 " apart) were performed and recorded for each sample.

\subsection{Preparation of "Mashed Banana Samples"}

A random mixture of banana fingers from the top, middle and bottom of the bunch were picked, washed and peeled. Mashed bananas were prepared by taking peeled bananas and steaming them in the middle chamber of a 3 Tier Food Steamer (HS6000, Black \& Decker, China) for 30 min before being mashed. Mashing was done by taking steamed bananas and wrapping them into two heat-softened banana leaves. They were wrapped further in another layer of a 30 micron plastic bag to enhance pressing and mashing. Banana samples were then hand-mashed by squeezing and pressing 30 times to produce the mashed bananas. The plastic bag was removed and the mashed bananas left wrapped in banana leaves, returned to the steamer and steamed for a further 20 min before taking the first sample. Samples of mashed bananas were placed in container cups $(4 \mathrm{x} 4.5 \mathrm{~cm})$ formed using aluminium foil and taken to the texture analyzer for analysis.

\subsection{Preparation of Cooled Bananas}

In order to determine the effect of cooling on textural hardness of cooked bananas, banana samples were taken at the end of each cooking treatment, placed on a table and left in the open air at room temperature to cool for 1 and $4 \mathrm{~h}$ after which textural hardness was measured, respectively.

\subsection{Determination of Textural Hardness}

Analysis of textural hardness was performed using a Texture Analyzer (TA.XT plus stable micro-systems, Surrey, UK) according to (Yang et al., 2007; Setiady, Tang, Younce, Swanson, \& Clary, 2009 and Kaur, Singh, Sodhi, \& Rana, 2009) with modifications. The measurement was performed by penetration using a penetration probe or rig (6 mm diameter). The Texture Analyzer was set in Return-to-start mode with the following test settings:penetration distance into the sample $-20 \mathrm{~mm}$, pre-test speed $-1.0 \mathrm{~mm} / \mathrm{s}$, test speed into sample $-2.0 \mathrm{~mm} / \mathrm{s}$, post-test speed $-10 \mathrm{~mm} / \mathrm{s}$, a trigger force of $0.049 \mathrm{~N}$ and calibrated using a $2 \mathrm{~kg}$ load cell. Banana samples were positioned in the middle of the Texture Analyzer platform and commanded to start. The measurements were performed in duplicate using two independent samples. The profile of the force in form of texture curves was monitored on a Personal Computer (PC) interfaced to the Texture Analyzer and the force needed to fracture or penetrate the banana sample was recorded as the first peak under the force-time curves and was taken as the hardness (N) (Jha et al., 2010a; Bagaud, Chillet, Beaute, \& Dubois, 2006).

\subsection{Statistical Analysis}

Data were analyzed using IBM SPSS package (version 23). Means were separated by group and were analyzed 
using one-way Analysis of Variance (ANOVA) using Fisher's Least Significant Difference (LSD) as well as Turkey's procedures and means were tested for homogeneity of variance. Other statistical procedures used include comparing means using the Paired Samples T-Test to determine significant differences in texture of a pair of cooked and cooled banana samples depending on the treatment. Significant differences were determined at $\mathrm{P}<0.05$.

\section{Results}

\subsection{Introduction}

The fourteen banana cultivars used in this study were categorized according to their usual consumption mode: as 'juice bananas' because they are used for juice production when ripe, and as "cooking bananas" because they are cooked before being consumed (Price, 1999). Bananas were drawn from clone sets as classified by Karamura (1998) in order to determine if there would be a relationship between and within the clone sets.

Cooking banana samples were subjected to dry matter and pulp-to-peel ratio evaluation which were monitored until their values were relatively constant, an indication of optimum maturity. At this stage, bananas are manufacturing little or no additional food reserves. All bananas had expressed signs of maturity i.e. fruit angles were filled in completely, the top leaves had dried, the colour of banana finger had changed from deep green to light green (Amin, Hossain, Rahim, \& Uddin, 2015) and the flesh of the pulp was orange-yellow for all cooking bananas. The banana fingers had reached relatively good size in comparison with the normal size of that particular banana cultivar basing on information from the on-farm guide at Kawanda Agricultural Research Institute (KARI). The edges of the banana fingers were fully round without being sharp (Amin et al., 2015). None of the bananas had reached the ripening stage. The stage of maturation at which the fruit is harvested influences the storage life, eating quality of bananas and may also affect texture. Bananas which are not fully mature have less appealing pulp and normally harder than mature ones when cooked. Every fruit attains full characteristics such as flavour, taste and colour during storage if it was harvested at optimum maturity (Amin et al., 2015). Bunches of bananas are harvested when the fruits are fully developed, that is, $75 \%$ matured, the angles are becoming less prominent and the fruits on the top are changing to light green; and the flower remnants (styles) are easily rubbed off the tips.

\subsection{Textural Hardness of the Raw Cooking and Juice Bananas}

Textural hardness of raw green unripe juice bananas ranged between $36.1 \mathrm{~N}$ and $42.43 \mathrm{~N}$ and was significantly higher $(\mathrm{P}<0.05)$ than that of raw green unripe cooking bananas which ranged from $22.30 \mathrm{~N}$ to $26.72 \mathrm{~N}$ (Table 1 ). For juice bananas, Ndiizi was significantly harder than both Kayinja and Kisubi. The hardness of both Kisubi and Ndiizi which belong to the Ney Poovan $\mathrm{AB}$ clone set varied significantly $(\mathrm{P}<0.05)$. There was no pronounced difference between Kisubi of Ney Poovan AB and Kayinja of the Bluggoes ABB clone sets. Data of cooking bananas indicates that Kibuzi was softer while Nakabululu and Kisansa were harder than the rest of the cooking banana cultivars. Hardness of Namande, Nakawere and Namweezi of the Nfuuka clone set was not significantly different $(\mathrm{P}<0.05)$. In the Nakitembe clone set, Nakitembe variety was harder than both Nakyetengu and Kibuzi. Cultivars under Musakala and Nakabululu clone sets were the hardest and there was no pronounced difference in hardness between cultivars in the Musakala as well as Nakabululu clone sets.

Table 1. Textural hardness of selected raw green unripe indigenous cooking and juice bananas from Uganda

\begin{tabular}{|c|c|c|c|}
\hline Local name of cultivar & **Synonym & Clone set & Textural hardness $(\mathrm{N})$ \\
\hline \multicolumn{4}{|l|}{ Cooking banana cultivars } \\
\hline Namande & NAMD & Nfuuka & $23.81^{\mathrm{abc}} \pm 3.19$ \\
\hline Nakawere & NAKW & Nfuuka & $22.81^{\mathrm{ab}} \pm 1.98$ \\
\hline Namweezi & NAMZ & Nfunka & $22.80^{\mathrm{ab}} \pm 1.97$ \\
\hline Nakitembe & NAKT & Nakitembe & $25.40^{\mathrm{abc}} \pm 1.49$ \\
\hline Nakyetengu & NAKY & Nakitembe & $22.53^{\mathrm{ab}} \pm 2.79$ \\
\hline Kibuzi & KIB & Nakitembe & $22.37^{\mathrm{a}} \pm 1.59$ \\
\hline Musakala & MUS & Musakala & $25.10^{\mathrm{abc}} \pm 1.00$ \\
\hline Mpologoma & MPO & Musakala & $23.65^{\mathrm{abc}} \pm 1.50$ \\
\hline Kisansa & KIS & Musakala & $26.00^{\mathrm{bc}} \pm 2.03$ \\
\hline Nakabululu & NAKB & Nakabululu & $26.36^{\mathrm{bc}} \pm 1.98$ \\
\hline Kazirakwe & KAZ & Nakabululu & $25.14^{\mathrm{abc}} \pm 1.15$ \\
\hline \multicolumn{4}{|l|}{ Juice banana cultivars } \\
\hline Kisubi & KISB & Ney Poovan AB & $36.17^{\mathrm{d}} \pm 1.45$ \\
\hline Ndiizi (apple banana) & NDI & Ney Poovan AB & $42.43^{\mathrm{e}} \pm 3.73$ \\
\hline Kayinja & KAY & Bluggoes ABB & $36.86^{\mathrm{d}} \pm 2.16$ \\
\hline
\end{tabular}

*Values with the same superscripts in the same column are not significantly different. 
$* \mathrm{~N}=$ Newtons, the penetration force as measured by the Texture analyzer (TA).

*Names and "clone set classification" adopted from Karamura (1998).

**Note: The synonyms for banana varieties in this Table are used in all sections hereafter.

\subsection{Influence of Boiling, Steaming and Mashing on Textural Hardness}

All banana cultivars both cooking and juice were subjected to boiling, steaming and mashing treatments and sampled for hardness tests at 30, 50,70, 90, 110 and $130 \mathrm{~min}$. The boiling temperature ranged between 97 to $98^{\circ} \mathrm{C}$ while that of steaming ranged between 98 to $99^{\circ} \mathrm{C}$. In general, boiling caused a rapid decrease in textural hardness of all bananas. Juice bananas were significantly harder than cooking bananas $(\mathrm{P}<0.05)$ (Table 2a). For juice bananas, boiling for 30 min caused a rapid decrease in hardness by average $81 \%$ and by $130 \mathrm{~min}$, hardness had decreased by $92 \%$. In general, KAY was harder than both KISB and NDI and their hardness decreased significantly with cooking time $(\mathrm{P}<0.05)$. For instance, hardness of KAY, KISB and NDI decreased from $8.89 \mathrm{~N}$, $6.58 \mathrm{~N}$, and $6.29 \mathrm{~N}$ at $30 \mathrm{~min}$ to $4.90 \mathrm{~N}, 2.67 \mathrm{~N}$ and $1.38 \mathrm{~N}$ at $130 \mathrm{~min}$. For cooking bananas, boiling for $30 \mathrm{~min}$ also caused a rapid decrease in textural hardness by average $96.2 \%$ and by $130 \mathrm{~min}$, hardness had decreased by average $98 \%$. Between 30 and 130 min of boiling, the decrease in hardness of most cooking bananas was not significant ( $\mathrm{P}>0.05$; Table 2a). However, NAKB, KAZ and NAKT experienced a significant decrease in hardness with increasing cooking time $(\mathrm{P}<0.05)$ and were harder than other varieties. On the other hand, KIB, NAMZ and NAKW maintained the lowest hardness. KIB was the softest while KAZ was the hardest.

Similarly, steaming caused a rapid decrease in hardness for both cooking and juice bananas within the first 30 min followed by a slow gradual decrease until $130 \mathrm{~min}$ (Table 2b). Juice bananas were still generally harder than cooking bananas. In the first 30 min of steaming, hardness of juice bananas decreased rapidly by $73.9 \%$ and by $130 \mathrm{~min}$, it had decreased by $90.6 \%$. Hardness of all three juice bananas significantly decreased between 30 and 70 min $(\mathrm{P}<0.05)$ but further decrease was not significant $(\mathrm{P}>0.05)$ except for KAY. In general, KAY was significantly harder than both KISB and NDI $(\mathrm{p}<0.05)$. Hardness of cooking bananas also decreased rapidly by $90.3 \%$ within $30 \mathrm{~min}$ of steaming and by $130 \mathrm{~min}$, it had decreased by $96.7 \%$. Hardness of almost all cultivars significantly decreased between 30 and $70 \mathrm{~min}$ of steaming $(\mathrm{P}<0.05)$ but further decrease was not significant ( $P>0.05$; Table 2b). Again, hardness of KIB was the lowest while that of NAKB and KAZ was the highest.

Mashed bananas were also cooked by steaming. In general, hardness of both "mashed" cooking and juice bananas decreased between 50 and $130 \mathrm{~min}$ of steaming although the decrease was not significant $(\mathrm{P}>0.05)$ except for NDI (Table 2c). Mashed juice bananas were still significantly harder than mashed cooking bananas throughout cooking $(\mathrm{P}<0.05)$. For juice bananas, hardness decreased by $92.50 \%$ by $130 \mathrm{~min}$ of steaming. KAY was still significantly harder than both KISB and NDI. For cooking bananas, hardness decreased by $97.62 \%$ by $130 \mathrm{~min}$ of steaming. Once again, KIB still had the lowest level of hardness while KAZ had the highest level of hardness. These results indicate that hardness of mashed bananas was intermediate between that of boiled and steamed bananas.

Table2a. Changes in textural hardness of selected indigenous cooking and juice banana cultivars during "boiling"

\begin{tabular}{|c|c|c|c|c|c|c|}
\hline \multirow[b]{3}{*}{ Cooking time (min) } & \multicolumn{6}{|c|}{ Cooking treatment } \\
\hline & \multicolumn{6}{|c|}{ Boiling } \\
\hline & 30 & 50 & 70 & 90 & 110 & 130 \\
\hline \multicolumn{7}{|c|}{ Cooking banana cultivars } \\
\hline NAMD & $0.64_{\mathrm{f}}^{\mathrm{f}} \pm 0.28$ & $0.62_{\mathrm{f}}^{\mathrm{f}} \pm 0.19$ & $0.36_{\mathrm{f}}^{\mathrm{f}} \pm 0.18$ & $0.43_{\mathrm{f}}^{\mathrm{f}} \pm 0.12$ & $0.35_{\mathrm{f}}^{\mathrm{f}} \pm 0.14$ & $0.38_{\mathrm{g}}{ }^{\mathrm{f}} \pm 0.10$ \\
\hline NAKW & $0.69_{\mathrm{f}}^{\mathrm{f}} \pm 0.18$ & $0.47_{\mathrm{f}}^{\mathrm{f}} \pm 0.18$ & $0.47_{\mathrm{f}}^{\mathrm{f}} \pm 0.10$ & $0.41_{\mathrm{f}}^{\mathrm{f}} \pm 0.17$ & $0.34_{\mathrm{f}}^{\mathrm{f}} \pm 0.07$ & $0.35_{\mathrm{g}}^{\mathrm{f}} \pm 0.11$ \\
\hline NAMZ & $0.5_{\mathrm{f}}^{\mathrm{f}} \pm 0.11$ & $0.7_{\mathrm{f}}^{\mathrm{f}} \pm 0.23$ & $0.52_{\mathrm{f}}^{\mathrm{f}} \pm 0.20$ & $0.39_{\mathrm{f}}^{\mathrm{f}} \pm 0.08$ & $0.33_{\mathrm{f}}^{\mathrm{f}} \pm 0.04$ & $0.37_{\mathrm{g}}^{\mathrm{f}} \pm 0.10$ \\
\hline NAKT & $1.08_{\mathrm{f}}^{\mathrm{g}} \pm 0.24$ & $0.94_{\mathrm{f}}^{\mathrm{fg}} \pm 0.09$ & $0.77_{\mathrm{f}}^{\mathrm{fg}} \pm 0.12$ & $0.65_{\mathrm{f}}^{\mathrm{fg}_{ \pm}} \pm 0.09$ & $0.62_{\mathrm{f}}^{\mathrm{fg}} \pm 0.10$ & $0.56_{\mathrm{g}}^{\mathrm{f}} \pm 0.30$ \\
\hline NAKY & $0.65_{\mathrm{f}}^{\mathrm{f}} \pm 0.19$ & $0.5_{\mathrm{f}}^{\mathrm{f}} \pm 0.21$ & $0.45_{\mathrm{f}}^{\mathrm{f}} \pm 0.12$ & $0.42_{\mathrm{f}}^{\mathrm{f}} \pm 0.11$ & $0.42_{\mathrm{f}}^{\mathrm{f}} \pm 0.08$ & $0.35_{\mathrm{g}}^{\mathrm{f}} \pm 0.12$ \\
\hline KIB & $0.56_{\mathrm{f}}^{\mathrm{f}} \pm 0.14$ & $0.5_{\mathrm{f}}^{\mathrm{f}} \pm 0.27$ & $0.43_{\mathrm{f}}^{\mathrm{f}} \pm 0.15$ & $0.42_{\mathrm{f}}^{\mathrm{f}} \pm 0.10$ & $0.38_{\mathrm{f}}^{\mathrm{f}} \pm 0.21$ & $0.34_{\mathrm{f}}^{\mathrm{f}} \pm 0.20$ \\
\hline MUS & $1.1_{\mathrm{f}}^{\mathrm{g}} \pm 0.42$ & $0.99_{\mathrm{f}}{ }^{\mathrm{fg}} \pm 0.19$ & $0.64_{\mathrm{f}}^{\mathrm{f}} \pm 0.14$ & $0.55_{\mathrm{f}}^{\mathrm{f}} \pm 0.13$ & $0.54_{f}^{f} \pm 0.24$ & $0.52_{\mathrm{g}}^{\mathrm{f}} \pm 0.15$ \\
\hline MPO & $0.68_{\mathrm{f}}^{\mathrm{f}} \pm 0.13$ & $0.64_{\mathrm{f}}^{\mathrm{f}} \pm 0.21$ & $0.58_{\mathrm{f}}^{\mathrm{f}} \pm 0.17$ & $0.54_{\mathrm{f}}^{\mathrm{f}} \pm 0.11$ & $0.43_{\mathrm{f}}^{\mathrm{f}} \pm 0.18$ & $0.35_{\mathrm{g}}^{\mathrm{f}} \pm 0.12$ \\
\hline KIS & $1.11_{\mathrm{f}}^{\mathrm{f}} \pm 0.47$ & $1.07_{\mathrm{f}}^{\mathrm{f}} \pm 0.50$ & $0.87_{\mathrm{f}}^{\mathrm{f}} \pm 0.22$ & $0.69_{\mathrm{f}}^{\mathrm{f}} \pm 0.26$ & $0.73_{\mathrm{g}}^{\mathrm{f}} \pm 0.29$ & $0.54_{\mathrm{g}}^{\mathrm{f}} \pm 0.22$ \\
\hline NAKB & $1.47_{\mathrm{f}}^{\mathrm{h}} \pm 0.23$ & $1.14_{\mathrm{f}}^{\mathrm{gh}} \pm 0.27$ & $0.83_{\mathrm{f}}^{\mathrm{fg}} \pm 0.26$ & $0.82_{\mathrm{g}}{ }^{\mathrm{fg}} \pm 0.26$ & $0.69_{\mathrm{g}}^{\mathrm{fg}} \pm 0.16$ & $0.51_{\mathrm{g}}^{\mathrm{f}} \pm 0.16$ \\
\hline KAZ & $1.67_{\mathrm{f}}^{\mathrm{h}} \pm 0.17$ & $1.13_{\mathrm{f}}^{\mathrm{gh}} \pm 0.5$ & $1.03_{\mathrm{f}}^{\mathrm{gh}} \pm 0.16$ & $0.98^{\mathrm{gh}} \pm 0.14$ & $0.89 \mathrm{~g}^{\mathrm{g}} \pm 0.25$ & $0.68_{\mathrm{g}}^{\mathrm{g}} \pm 0.10$ \\
\hline \multicolumn{7}{|c|}{ Juice banana cultivars } \\
\hline KISB & $6.58_{\mathrm{g}}{ }^{\mathrm{k}} \pm 0.87$ & $4.82_{\mathrm{g}}^{\mathrm{jk}} \pm 0.97$ & $3.77_{\mathrm{g}}^{\mathrm{ij}} \pm 0.69$ & $3.49_{\mathrm{h}}{ }^{\mathrm{ij}} \pm 0.85$ & $2.73_{\mathrm{h}}{ }^{\mathrm{i}} \pm 0.44$ & $2.67_{\mathrm{h}}^{\mathrm{i}} \pm 0.49$ \\
\hline NDI & $6.29_{\mathrm{g}}^{\mathrm{k}} \pm 1.48$ & $4.14_{\mathrm{g}}^{\mathrm{jk}} \pm 0.67$ & $2.79_{\mathrm{g}}^{\mathrm{ij}} \pm 0.68$ & $1.74_{\mathrm{g}}{ }^{\mathrm{i}} \pm 0.43$ & $1.499_{\mathrm{g}}^{\mathrm{i}} \pm 0.43$ & $1.38_{\mathrm{g}}{ }^{\mathrm{i}} \pm 0.67$ \\
\hline KAY & $8.89_{\mathrm{h}}^{\mathrm{m}} \pm 1.75$ & $6.79_{\mathrm{h}}^{\mathrm{kl}} \pm 0.74$ & $6.5_{\mathrm{h}}^{\mathrm{kl}} \pm 0.84$ & $6.22_{\mathrm{i}}^{\mathrm{k}} \pm 0.52$ & $4.97_{i}^{j \mathrm{j}} \pm 0.59$ & $4.9_{i}^{j \mathrm{k}} \pm 0.83$ \\
\hline
\end{tabular}

Values with different superscripts along rows or subscripts along columns are significantly different. 
Table2b. Changes in textural hardness of selected indigenous cooking and juice banana cultivars during "steaming"

\begin{tabular}{|c|c|c|c|c|c|c|}
\hline \multirow{3}{*}{ Cooking time (min) } & \multicolumn{6}{|c|}{ Cooking treatment } \\
\hline & \multicolumn{6}{|c|}{ Steaming } \\
\hline & 30 & 50 & 70 & 90 & 110 & 130 \\
\hline \multicolumn{7}{|c|}{ Cooking banana cultivars } \\
\hline NAMD & $1.52_{\mathrm{fg}}{ }^{\mathrm{f}} \pm 0.29$ & $1.18_{\mathrm{f}}^{\mathrm{fg}} \pm 0.24$ & $0.99_{\mathrm{f}}^{\mathrm{fg}} \pm 0.11$ & $0.95_{\mathrm{f}}^{\mathrm{g}} \pm 0.19$ & $0.92_{\mathrm{f}}{ }^{\mathrm{g}} \pm 0.11$ & $0.78_{\mathrm{f}}{ }^{\mathrm{g}} \pm 0.17$ \\
\hline NAKW & $1.82_{\mathrm{fg}}{ }^{\mathrm{f}} \pm 0.45$ & $1.19_{\mathrm{f}}^{\mathrm{fg}} \pm 0.16$ & $0.97_{\mathrm{f}}^{g} \pm 0.17$ & $0.91_{\mathrm{f}}^{\mathrm{g}} \pm 0.26$ & $0.85_{\mathrm{f}}^{\mathrm{g}} \pm 0.14$ & $0.61_{\mathrm{f}}{ }^{\mathrm{g}} \pm 0.11$ \\
\hline NAMZ & $2.73_{\mathrm{fg}}{ }^{\mathrm{f}} \pm 0.74$ & $1.1_{\mathrm{f}}^{\mathrm{g}} \pm 0.15$ & $1.05_{\mathrm{f}}^{\mathrm{g}} \pm 0.13$ & $1.04_{\mathrm{f}}^{\mathrm{g}} \pm 0.08$ & $0.92_{\mathrm{f}}{ }^{\mathrm{g}} \pm 0.18$ & $0.74_{\mathrm{f}}^{\mathrm{g}} \pm 0.15$ \\
\hline NAKT & $1.48_{\mathrm{fg}}^{\mathrm{f}} \pm 0.17$ & $1.18_{\mathrm{f}}^{\mathrm{fg}} \pm 0.18$ & $0.98_{\mathrm{f}}^{\mathrm{fg}} \pm 0.33$ & $0.89_{\mathrm{f}}^{\mathrm{g}} \pm 0.12$ & $0.94_{\mathrm{f}}^{\mathrm{fg}} \pm 0.12$ & $0.76_{\mathrm{f}}^{\mathrm{g}} \pm 0.19$ \\
\hline NAKY & $1.82_{\mathrm{fg}}{ }^{\mathrm{f}} \pm 0.23$ & $1.26_{\mathrm{f}}^{\mathrm{fg}} \pm 0.18$ & $1.03_{\mathrm{f}}{ }^{\mathrm{g}} \pm 0.34$ & $0.97_{\mathrm{f}}^{\mathrm{g}} \pm 0.25$ & $0.95_{\mathrm{f}} \mathrm{g}_{ \pm} \pm 0.33$ & $0.8_{\mathrm{f}}^{\mathrm{g}} \pm 0.09$ \\
\hline KIB & $1.34_{\mathrm{f}}^{\mathrm{f}} \pm 0.18$ & $1.05_{\mathrm{f}}^{\mathrm{fg}} \pm 0.16$ & $0.72_{\mathrm{f}}^{\mathrm{gh}} \pm 0.19$ & $0.70_{\mathrm{f}}^{\mathrm{gh}} \pm 0.14$ & $0.64_{\mathrm{f}}^{\mathrm{h}} \pm 0.05$ & $0.60_{\mathrm{f}}^{\mathrm{h}} \pm 0.12$ \\
\hline MUS & $3.95_{\mathrm{g}}^{\mathrm{f}} \pm 0.24$ & $1.29_{\mathrm{f}}^{\mathrm{g}} \pm 0.22$ & $1.13_{\mathrm{f}}^{\mathrm{gh}} \pm 0.16$ & $0.74_{\mathrm{f}}^{\mathrm{h}} \pm 0.12$ & $0.73_{\mathrm{f}}^{\mathrm{h}} \pm 0.12$ & $0.72_{\mathrm{f}}^{\mathrm{h}} \pm 0.15$ \\
\hline MPO & $1.85_{\mathrm{fg}} \mathrm{f}^{\mathrm{f}} \pm 0.35$ & $1.1_{\mathrm{f}}^{\mathrm{g}} \pm 0.27$ & $1.05_{\mathrm{f}}^{\mathrm{g}} \pm 0.21$ & $0.72_{\mathrm{f}} \mathrm{g}^{\mathrm{g}} \pm 0.11$ & $0.71_{\mathrm{f}} \mathrm{g}_{ \pm 0} \mathrm{~g} .12$ & $0.6_{\mathrm{f}}^{\mathrm{g}} \pm 0.06$ \\
\hline KIS & $3.14_{\mathrm{g}}^{\mathrm{f}} \pm 0.70$ & $1.82_{\mathrm{f}} \mathrm{g}_{ \pm} \pm 0.62$ & $1.13_{\mathrm{f}}^{\mathrm{g}} \pm 0.43$ & $1.10_{\mathrm{f}}^{\mathrm{g}} \pm 0.19$ & $1.04_{\mathrm{f}}^{\mathrm{g}} \pm 0.29$ & $0.97_{\mathrm{f}}^{\mathrm{g}} \pm 0.30$ \\
\hline NAKB & $3.67_{\mathrm{g}}^{\mathrm{f}} \pm 0.76$ & $1.65_{\mathrm{f}}^{\mathrm{g}} \pm 0.33$ & $1.36_{\mathrm{fg}}{ }^{\mathrm{g}} \pm 0.23$ & $1.27_{\mathrm{f}}^{\mathrm{g}} \pm 0.26$ & $1.19_{\mathrm{f}}^{\mathrm{g}} \pm 0.18$ & $1.05_{\mathrm{f}}^{\mathrm{g}} \pm 0.14$ \\
\hline KAZ & $2.5_{\mathrm{fg}}{ }^{\mathrm{f}} \pm 0.33$ & $1.76_{\mathrm{f}}^{\mathrm{fg}} \pm 0.30$ & $1.47_{\mathrm{fg}}{ }^{\mathrm{g}} \pm 0.28$ & $1.44_{\mathrm{f}}^{\mathrm{g}} \pm 0.45$ & $1.45_{\mathrm{f}}^{\mathrm{g}} \pm 0.19$ & $1.24_{\mathrm{f}}^{\mathrm{g}} \pm 0.15$ \\
\hline \multicolumn{7}{|c|}{ Juice banana cultivars } \\
\hline KISB & $6.93_{\mathrm{h}}{ }^{\mathrm{f}} \pm 1.34$ & $4.8_{\mathrm{f}}^{\mathrm{fg}} \pm 1.36$ & $3.97 \mathrm{~g}^{\mathrm{g}} \pm 0.60$ & $3.77 \mathrm{~g}^{\mathrm{g}} \pm 0.22$ & $3.18_{\mathrm{g}}^{\mathrm{g}} \pm 0.37$ & $2.95_{\mathrm{g}}^{\mathrm{g}} \pm 0.49$ \\
\hline NDI & $9.91_{i}^{\mathrm{f}} \pm 1.87$ & $4.58_{\mathrm{f}}^{\mathrm{g}} \pm 1.43$ & $4.13^{\mathrm{g}} \pm 0.78$ & $3.00_{\mathrm{g}}^{\mathrm{g}} \pm 1.39$ & $2.91_{\mathrm{g}}^{\mathrm{g}} \pm 0.47$ & $2.67^{\mathrm{g}} \pm 0.24$ \\
\hline KAY & $13.3_{\mathrm{j}}^{\mathrm{f}} \pm 1.75$ & $10.0_{\mathrm{f}}^{\mathrm{fg}} \pm 1.65$ & $8.19^{\mathrm{gh}} \pm 3.06$ & $6.40_{\mathrm{h}}^{\mathrm{gh}} \pm 1.00$ & $6.5_{\mathrm{h}}^{\mathrm{gh}} 8 \pm 0.95$ & $5.29_{\mathrm{h}}^{\mathrm{i}} \pm 0.56$ \\
\hline
\end{tabular}

Values with different superscripts along rows or subscripts along columns are significantly different.

Table2c. Changes in textural hardness of selected indigenous cooking and juice banana cultivars subjected to "mashing" during steaming

\begin{tabular}{|c|c|c|c|c|c|c|}
\hline \multirow{3}{*}{ Cooking time (min) } & \multicolumn{6}{|c|}{ Cooking treatment } \\
\hline & \multicolumn{6}{|c|}{ "Mashed bananas cooked by steaming" } \\
\hline & 30 & 50 & 70 & 90 & 110 & 130 \\
\hline \multicolumn{7}{|c|}{ Cooking banana cultivars } \\
\hline NAMD & - & $0.76_{\mathrm{f}}^{\mathrm{f}} \pm 0.10$ & $0.74_{\mathrm{f}}^{\mathrm{f}} \pm 0.07$ & $0.70_{\mathrm{f}}^{\mathrm{f}} \pm 0.13$ & $0.69_{\mathrm{f}}^{\mathrm{f}} \pm 0.06$ & $0.64_{\mathrm{f}}^{\mathrm{f}} \pm 0.16$ \\
\hline NAKW & - & $0.62_{\mathrm{f}}^{\mathrm{f}} \pm 0.11$ & $0.59_{\mathrm{f}}^{\mathrm{f}} \pm 0.14$ & $0.49_{\mathrm{f}}^{\mathrm{f}} \pm 0.05$ & $0.47_{\mathrm{f}}^{\mathrm{f}} \pm 0.05$ & $0.52_{\mathrm{f}}^{\mathrm{f}} \pm 0.03$ \\
\hline NAMZ & - & $0.76_{\mathrm{f}}^{\mathrm{f}} \pm 0.07$ & $0.62_{\mathrm{f}}^{\mathrm{fg}} \pm 0.06$ & $0.60_{\mathrm{f}}^{\mathrm{g}} \pm 0.04$ & $0.58_{\mathrm{f}}^{\mathrm{g}} \pm 0.02$ & $0.56_{f} \mathrm{~g}_{ \pm 0} \pm .09$ \\
\hline NAKT & - & $0.98_{\mathrm{fg}}{ }^{\mathrm{f}} \pm 0.15$ & $0.64_{f}^{g} \pm 0.16$ & $0.60_{\mathrm{f}}^{\mathrm{g}} \pm 0.12$ & $0.58_{\mathrm{f}}^{\mathrm{g}} \pm 0.07$ & $0.60_{f}^{g} \pm 0.11$ \\
\hline NAKY & - & $0.71_{\mathrm{f}}^{\mathrm{f}} \pm 0.05$ & $0.67_{\mathrm{f}}^{\mathrm{f}} \pm 0.08$ & $0.64_{\mathrm{f}}^{\mathrm{f}} \pm 0.18$ & $0.64_{\mathrm{f}}^{\mathrm{f}} \pm 0.11$ & $0.59_{\mathrm{f}}^{\mathrm{f}} \pm 0.07$ \\
\hline KIB & - & $0.60_{\mathrm{f}}^{\mathrm{f}} \pm 0.08$ & $0.54_{\mathrm{f}}^{\mathrm{fg}} \pm 0.09$ & $0.44_{\mathrm{f}}^{\mathrm{fg}} \pm 0.04$ & $0.45_{\mathrm{f}}^{\mathrm{fg}} \pm 0.03$ & $0.42_{\mathrm{f}}^{\mathrm{g}} \pm 0.05$ \\
\hline MUS & - & $0.71_{\mathrm{f}}^{\mathrm{f}} \pm 0.09$ & $0.63_{\mathrm{f}}^{\mathrm{f}} \pm 0.13$ & $0.65_{\mathrm{f}}^{\mathrm{f}} \pm 0.17$ & $0.61_{\mathrm{f}}^{\mathrm{f}} \pm 0.15$ & $0.48_{\mathrm{f}}^{\mathrm{f}} \pm 0.07$ \\
\hline MPO & - & $0.54_{\mathrm{f}}^{\mathrm{f}} \pm 0.05$ & $0.57_{\mathrm{f}}^{\mathrm{f}} \pm 0.09$ & $0.53_{\mathrm{f}}^{\mathrm{f}} \pm 0.07$ & $0.51_{\mathrm{f}}^{\mathrm{f}} \pm 0.07$ & $0.5_{\mathrm{f}}^{\mathrm{f}} \pm 0.06$ \\
\hline KIS & - & $0.74_{\mathrm{f}}^{\mathrm{f}} \pm 0.28$ & $0.52_{\mathrm{f}}^{\mathrm{f}} \pm 0.18$ & $0.55_{\mathrm{f}}^{\mathrm{f}} \pm 0.03$ & $0.55_{\mathrm{f}}^{\mathrm{f}} \pm 0.03$ & $0.54_{\mathrm{f}}^{\mathrm{f}} \pm 0.12$ \\
\hline NAKB & - & $0.77_{\mathrm{f}}^{\mathrm{f}} \pm 0.10$ & $0.69_{\mathrm{f}}^{\mathrm{f}} \pm 0.08$ & $0.65_{\mathrm{f}}^{\mathrm{f}} \pm 0.05$ & $0.61_{\mathrm{f}}^{\mathrm{f}} \pm 0.39$ & $0.61_{\mathrm{f}}^{\mathrm{f}} \pm 0.06$ \\
\hline KAZ & - & $1.17_{\mathrm{fg}}{ }^{\mathrm{f}} \pm 0.17$ & $0.96_{f}^{f} \pm 0.23$ & $0.86_{f}^{f} \pm 0.38$ & $0.85_{\mathrm{f}}^{\mathrm{f}} \pm 0.14$ & $0.88_{\mathrm{f}}^{\mathrm{f}} \pm 0.22$ \\
\hline \multicolumn{7}{|l|}{ Juice banana cultivars } \\
\hline KISB & - & $2.69_{\mathrm{fg}}^{\mathrm{f}} \pm 0.54$ & $2.82 \mathrm{~g} \pm 0.63$ & $2.61_{\mathrm{g}}^{\mathrm{f}} \pm 0.25$ & $2.14_{\mathrm{g}}^{\mathrm{f}} \pm 0.41$ & $2.12{ }_{\mathrm{g}}^{\mathrm{f}} \pm 0.34$ \\
\hline NDI & - & $4.41_{\mathrm{h}}^{\mathrm{f}} \pm 1.42$ & $2.54^{\mathrm{g}} \mathrm{g}_{ \pm 0}{ }^{\mathrm{g}}$ & $2.74^{\mathrm{g}} \mathrm{g}_{ \pm} \pm 0.68$ & $2.59_{\mathrm{g}}^{\mathrm{g}} \pm 0.84$ & $2.24_{\mathrm{g}}^{\mathrm{g}} \pm 0.20$ \\
\hline KAY & - & $4.96_{\mathrm{h}}^{\mathrm{f}} \pm 1.47$ & $4.34_{\mathrm{h}}{ }^{\mathrm{f}} \pm 2.20$ & $4.17_{\mathrm{h}}^{\mathrm{f}} \pm 0.16$ & $4.34_{\mathrm{h}}^{\mathrm{f}} \pm 1.99$ & $4.3_{\mathrm{h}} \mathrm{f}^{\mathrm{f}} \pm 0.56$ \\
\hline
\end{tabular}

Values with different superscripts along rows or subscripts along columns are significantly different.

\subsection{Influence of Cooking Time on Textural Hardness}

All banana samples were subjected to a cooking time ranging from 30 to $130 \mathrm{~min}$. The aim was to examine if extended cooking time leads to a significant reduction in textural hardness. Textural hardness of all banana cultivars decreased with cooking time from 30 to 130 min depending on cooking treatment. Hardness of cooking bananas at $30 \mathrm{~min}$ of boiling was not significantly different from hardness at $130 \mathrm{~min}$ of boiling (Table 2a). Hardness of cooking bananas at 30 min of boiling ranged between $0.5 \mathrm{~N}$ to $1.67 \mathrm{~N}$ while at 130 min of boiling, hardness had decreased in the range between $0.34 \mathrm{~N}$ and $0.68 \mathrm{~N}$. For juice bananas, hardness at 30 min of boiling was significantly higher than corresponding hardness at $130 \mathrm{~min}$ of boiling (Table 2a).On the other hand, hardness of all bananas steamed for 30 min was significantly higher than their corresponding hardness at 130 min of steaming $(\mathrm{P}<0.05)$ (Table $2 \mathrm{~b}$ and Figure 1$)$. The decrease in hardness of mashed bananas was not significantly related with increasing cooking time.

When boiled bananas were cooled and evaluated for hardness, all bananas boiled for 30 min were significantly harder than those boiled for 130 min (Figure 2) $(\mathrm{P}<0.05)$. For instance, the hardness of cooking bananas boiled 
for $30 \mathrm{~min}$ and allowed to cool ranged between $2.33 \mathrm{~N}$ (KIB) and $5.11 \mathrm{~N}$ (KAZ) while that of juice bananas ranged between $13.02 \mathrm{~N}$ (KISB) to $18.41 \mathrm{~N}$ (KAY). However, when the same bananas were boiled for $130 \mathrm{~min}$ and cooled, hardness of cooking bananas ranged between $1.21 \mathrm{~N}$ (KIB) and $4.02 \mathrm{~N}$ (NAKB) while that of juice bananas ranged between $9.36 \mathrm{~N}$ (KISB) and $12.65 \mathrm{~N}$ (KAY). This effect was more pronounced in steamed bananas than in boiled ones.

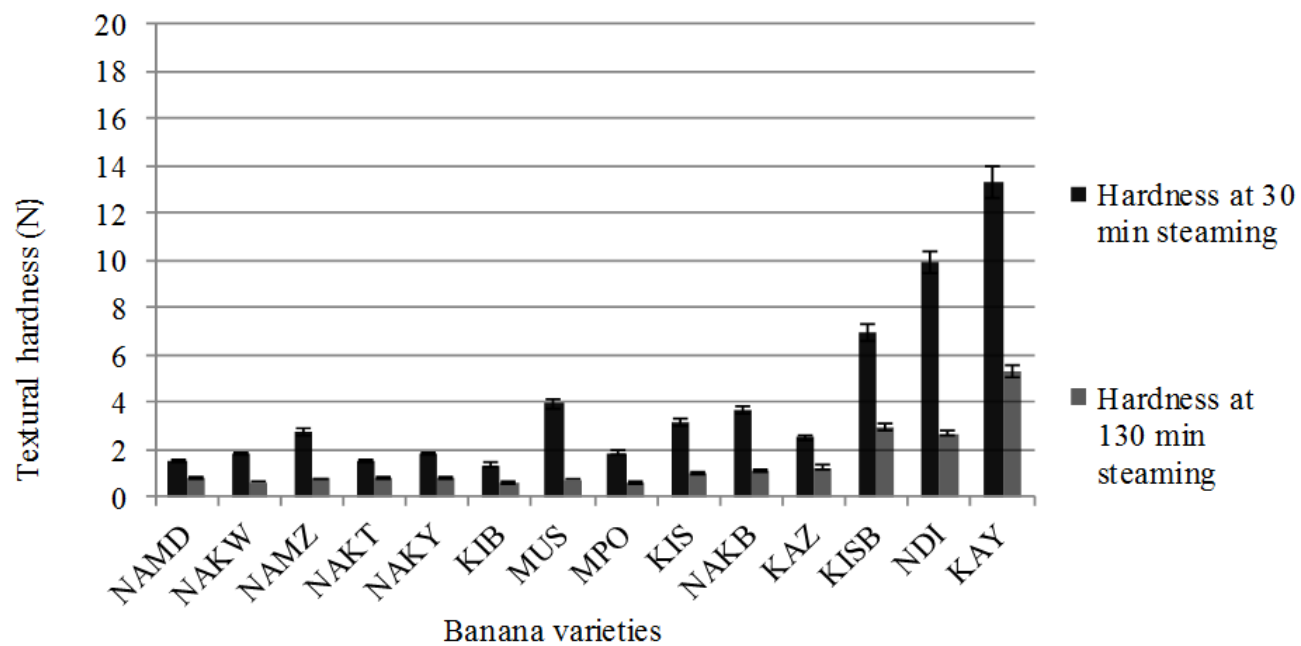

Figure 1. Comparison of textural hardness of bananas steamed for 30 and 130 min: The shorter bars represent hardness of bananas steamed for 130 min upon cooling while longer bars represent hardness of bananas steamed for 30 min upon cooling

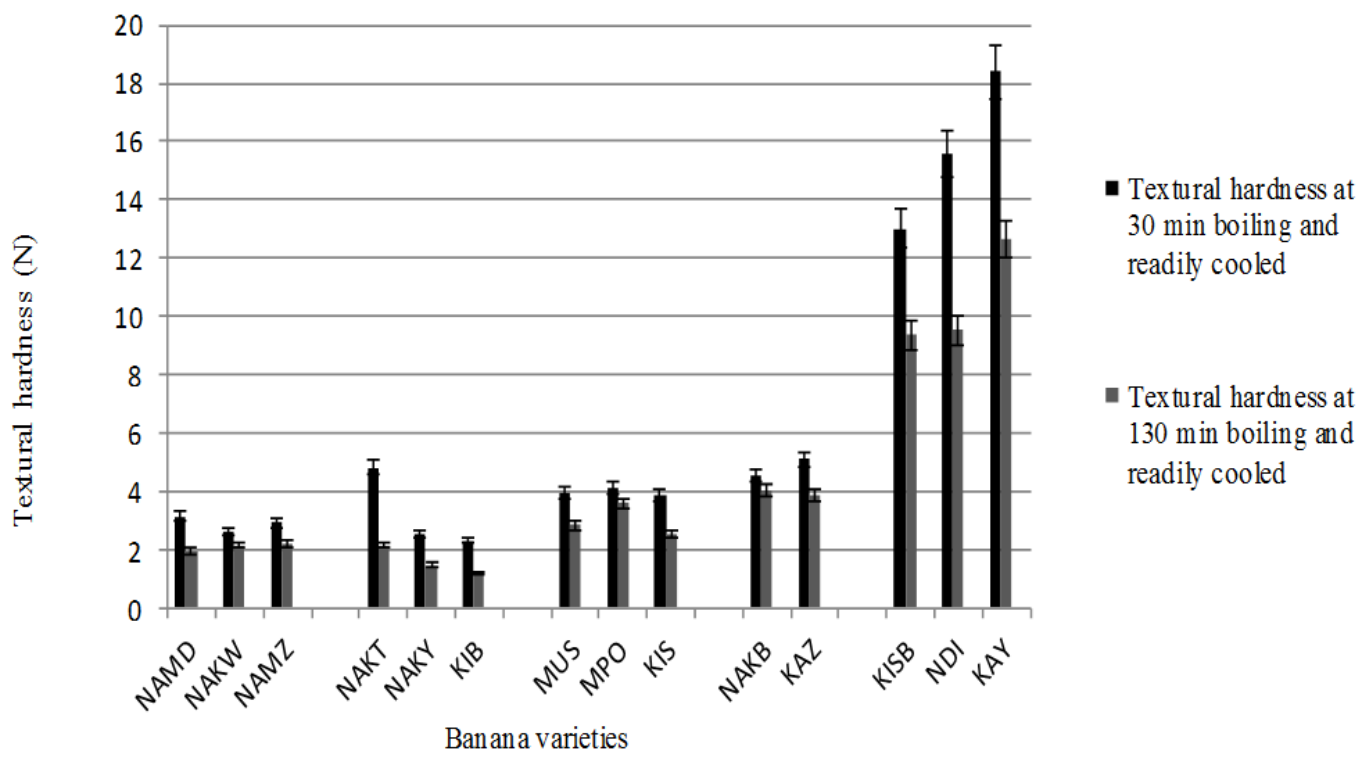

Figure 2. Comparison of textural hardness of bananas cooked for 30 and $130 \mathrm{~min}$ upon cooling. The shorter bars represent hardness of bananas steamed for 130 min upon cooling while longer bars represent hardness of bananas steamed for 30 min upon cooling

\subsection{Influence of Cooling on the Textural Hardness}

All banana cultivars were subjected to a cooling process in order to determine if cooked bananas would significantly harden upon cooling. Textural hardness was measured first at $1 \mathrm{~h}$ and after $4 \mathrm{~h}$ of cooling. For the first $1 \mathrm{~h}$ of cooling, samples were cooled in the open at room temperature. After $1 \mathrm{~h}$, the samples were placed in perforated plastic bags of 30 microns to allow thorough cooling without encouraging surface case-hardening that 
would result due to excessive loss of moisture from the surface.

Cooling significantly increased hardness of all banana cultivars $(\mathrm{P}<0.05)$ under all treatments. Juice bananas were significantly harder than cooking bananas in all cases $(\mathrm{P}<0.05)$ (Table 3$)$. In cooled form, hardness of boiled cooking bananas ranged from $1.04 \mathrm{~N}$ to $4.02 \mathrm{~N}$ while that of juice bananas ranged from $9.54 \mathrm{~N}$ to $12.65 \mathrm{~N}$. Hardness of steamed cooking bananas ranged between $2.97 \mathrm{~N}$ and $5.39 \mathrm{~N}$ while that of juice bananas ranged between $15.85 \mathrm{~N}$ and $18.33 \mathrm{~N}$ upon cooling. Hardness of cooled mashed cooking bananas ranged between 3.84 $\mathrm{N}$ to $6.89 \mathrm{~N}$ while that of juice bananas ranged between $14.41 \mathrm{~N}$ to $20.44 \mathrm{~N}$.

Overall, KAZ and NAKB were among the top three hardest cooking bananas in cooled as well as raw forms. KIB maintained the lowest textural hardness upon cooling under all cooking treatments (Table 3). For juice bananas, KAY was the hardest and KISB was the softest in cooled form.

Rate of textural hardening: All banana samples resulting from boiling, steaming and mashing treatments experienced a process of rapid textural hardening within the first $1 \mathrm{~h}$ of cooling, followed by a slow gradual increase in hardness (Figures 3, 4 and 5). The magnitude of hardening was influenced by the cooking time and treatment the bananas were subjected to. For example, when MUS was boiled for $30 \mathrm{~min}$ and cooled for $1 \mathrm{~h}$, the hardness increased rapidly from $1.10 \mathrm{~N}$ to $3.36 \mathrm{~N}$ (305\%). Further cooling increased the hardness to only $3.98 \mathrm{~N}$ (by a factor of 18.5\%). Whereas the hardness of MUS boiled for $130 \mathrm{~min}$ and cooled for $1 \mathrm{~h}$ experienced rapid hardening to $2.31 \mathrm{~N}$, further cooling for an extra $4 \mathrm{~h}$ only increased hardness to $2.85 \mathrm{~N}$. Steamed bananas experienced a higher rate of hardening in the first hour of cooling compared to both boiled and mashed ones. For example, MUS steamed for 130 min rapidly hardened to $3.27 \mathrm{~N}$ within the first hour of cooling. Further cooling for an extra $4 \mathrm{~h}$ only increased the hardness to $4.05 \mathrm{~N}$.

Results indicate that upon cooling, mashed bananas were generally harder than steamed or boiled bananas (Table $3)$.

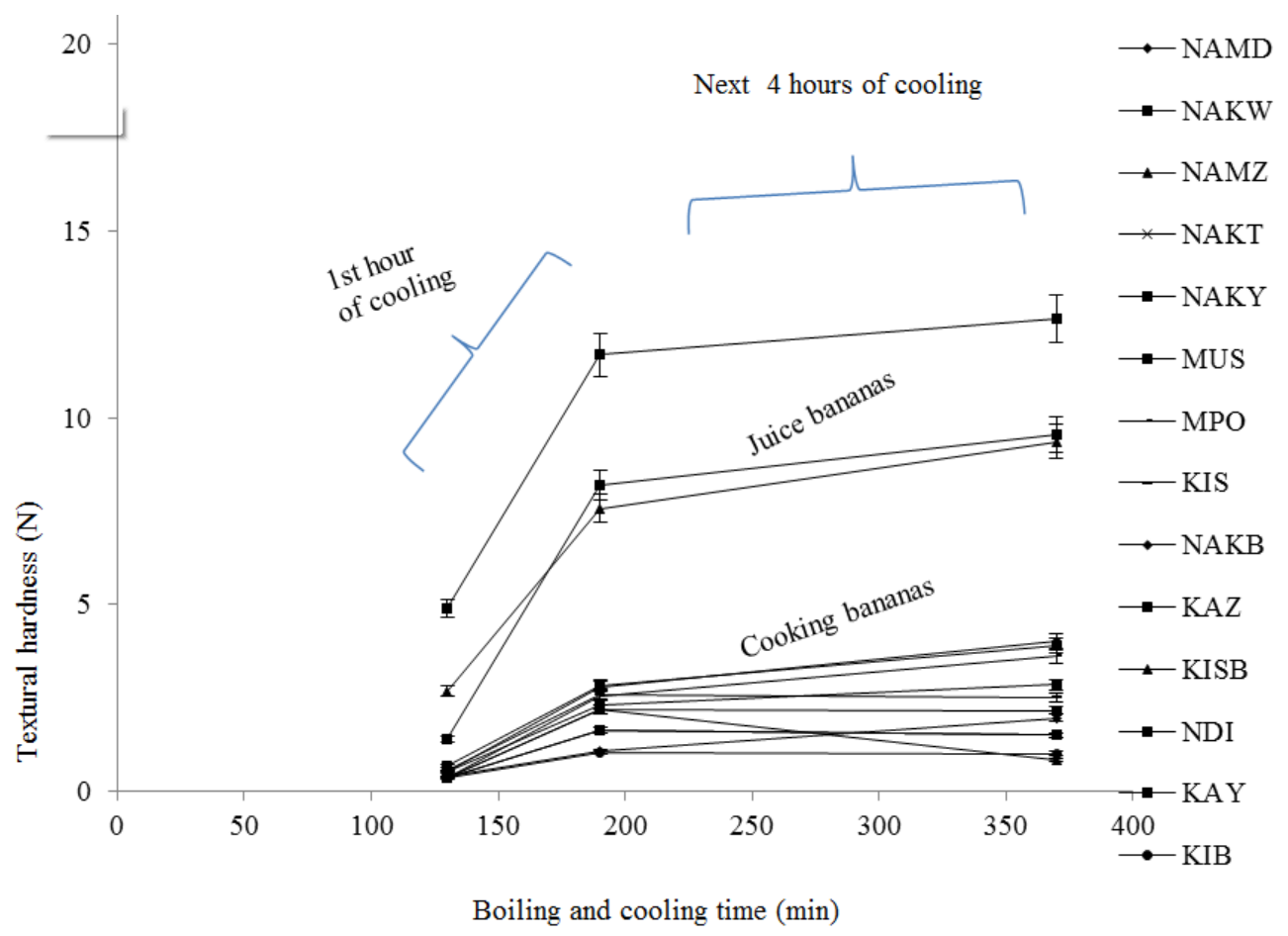

Figure 3. First order textural hardening kinetics occuring in boiled bananas during the first $1 \mathrm{~h}$, followed by slow gradual hardening in the next $4 \mathrm{~h}$, of cooling 


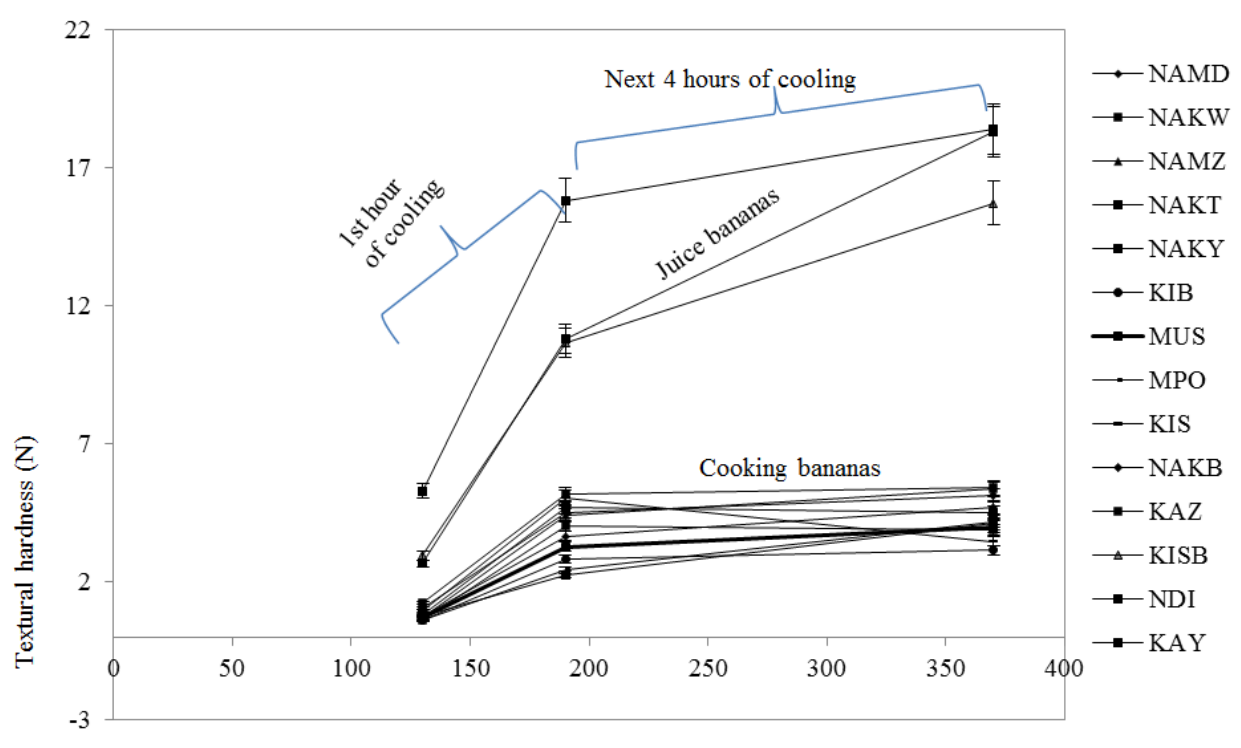

Steaming and cooling time $(\mathrm{min})$

Figure 4. First order textural hardening kinetics occuring in steamed bananas during the first $1 \mathrm{~h}$, followed by slow gradual hardening in the next $4 \mathrm{~h}$, of cooling

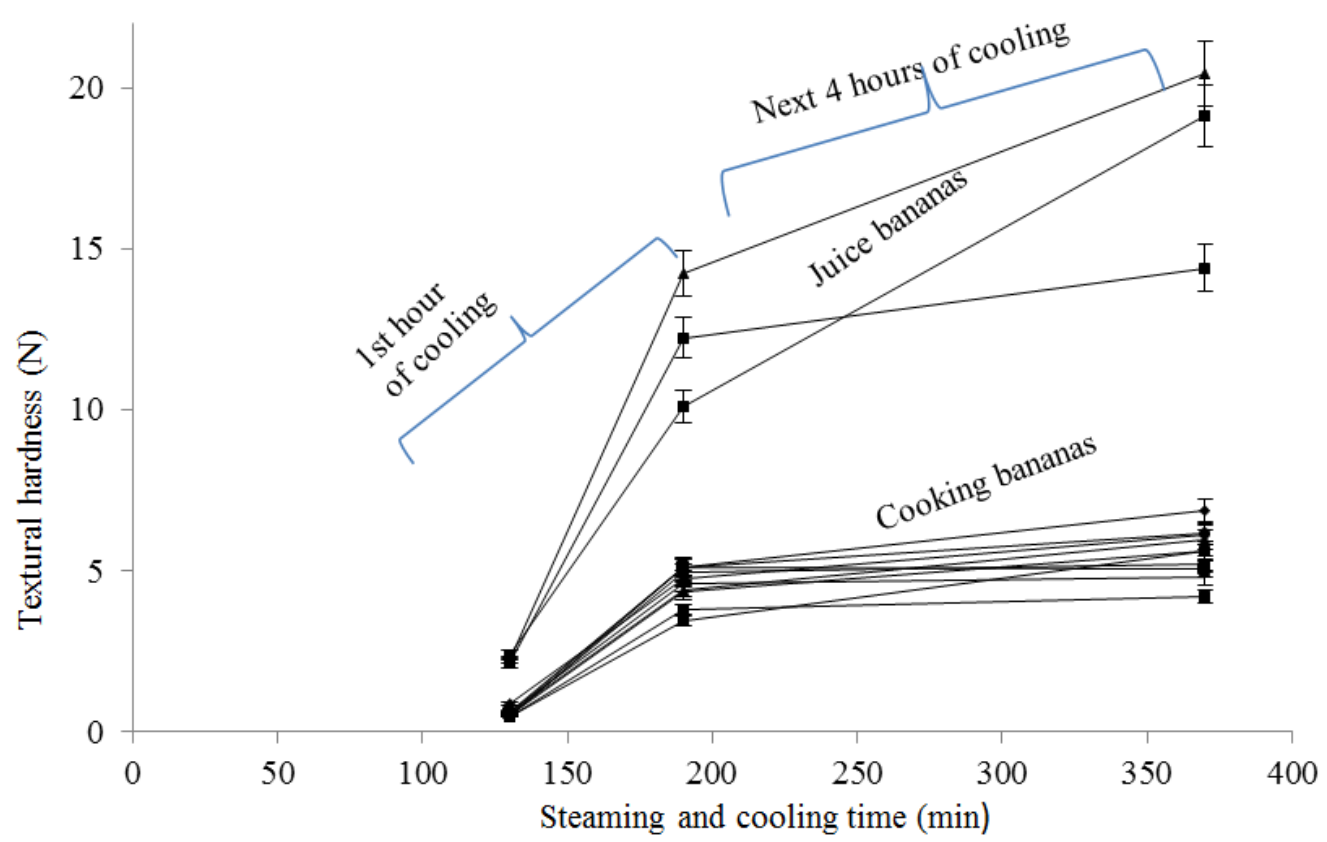

Figure 5. First order textural hardening kinetics occuring in mashed bananas during the first $1 \mathrm{~h}$, followed by slow gradual hardening in the next $4 \mathrm{~h}$, of cooling 
Table 3. Textural hardness of cooled bananas obtained from different cooking treatments

\begin{tabular}{|c|c|c|c|c|c|c|}
\hline \multirow{2}{*}{$\begin{array}{l}\text { Banana } \\
\text { varieties }\end{array}$} & \multicolumn{2}{|c|}{ BOILED BANANAS } & \multicolumn{2}{|c|}{ STEAMED BANANAS } & \multicolumn{2}{|c|}{ MASHED BANANAS } \\
\hline & $\begin{array}{l}\text { Hardness }(N) \text { at } \\
130 \text { min boiling }\end{array}$ & $\begin{array}{l}\text { Hardness }(N) \text { when } \\
\text { readily cooled }(4 h)\end{array}$ & $\begin{array}{l}\text { Hardness }(\mathrm{N}) \\
\text { at 130min } \\
\text { steaming } \\
\end{array}$ & $\begin{array}{lr}\text { Hardness } & (\mathrm{N}) \\
\text { when } & \text { readily } \\
\text { cooled }(4 \mathrm{~h}) & \\
\end{array}$ & $\begin{array}{l}\text { Hardness }(N) \text { at } \\
130 \mathrm{~min} \\
\text { steaming }\end{array}$ & $\begin{array}{lr}\text { Hardness } & (\mathrm{N}) \\
\text { when readily } \\
\text { cooled }(4 \mathrm{~h})\end{array}$ \\
\hline \multicolumn{7}{|c|}{ Cooking bananas } \\
\hline NAMD & $0.38 \pm 0.10^{\mathrm{a}}$ & $1.95 \pm 1.19^{\mathrm{b}}$ & $0.78 \pm 0.17^{\mathrm{a}}$ & $4.77 \pm 0.95^{b}$ & $0.70 \pm 0.16^{\mathrm{a}}$ & $6.89 \pm 0.53^{b}$ \\
\hline NAKW & $0.35 \pm 0.11^{\mathrm{a}}$ & $2.16 \pm 0.86^{\mathrm{b}}$ & $0.61 \pm 0.11^{\mathrm{a}}$ & $3.82 \pm 0.47^{\mathrm{b}}$ & $0.52 \pm 0.03^{\mathrm{a}}$ & $5.22 \pm 0.39^{b}$ \\
\hline NAMZ & $0.37 \pm 0.10^{\mathrm{a}}$ & $2.19 \pm 0.51^{\mathrm{b}}$ & $0.74 \pm 0.15^{\mathrm{a}}$ & $5.31 \pm 1.07^{\mathrm{b}}$ & $0.58 \pm 0.09^{\mathrm{a}}$ & $5.99 \pm 0.34^{\mathrm{b}}$ \\
\hline NAKT & $0.56 \pm 0.30^{\mathrm{a}}$ & $2.16 \pm 1.52^{\mathrm{b}}$ & $0.76 \pm 0.19^{\mathrm{a}}$ & $4.13 \pm 0.49^{b}$ & $0.60 \pm 0.12^{\mathrm{a}}$ & s.p \\
\hline NAKY & $0.35 \pm 0.12^{\mathrm{a}}$ & $1.49 \pm 0.36^{\mathrm{b}}$ & $0.80 \pm 0.09^{\mathrm{a}}$ & $4.48 \pm 0.62^{b}$ & $0.59 \pm 0.07^{\mathrm{a}}$ & $5.09 \pm 0.62^{\mathrm{b}}$ \\
\hline KIB & $0.34 \pm 0.20^{\mathrm{a}}$ & $1.04 \pm 0.17^{\mathrm{b}}$ & $0.60 \pm 0.12^{\mathrm{a}}$ & $2.97 \pm 0.50^{\mathrm{b}}$ & $0.49 \pm 0.05^{\mathrm{a}}$ & $3.84 \pm 0.36^{\mathrm{b}}$ \\
\hline MUS & $0.52 \pm 0.15^{\mathrm{a}}$ & $2.85 \pm 0.43^{\mathrm{b}}$ & $0.72 \pm 0.15^{\mathrm{a}}$ & $4.05 \pm 0.46^{\mathrm{b}}$ & $0.48 \pm 0.07^{\mathrm{a}}$ & $6.15 \pm 0.62^{b}$ \\
\hline MPO & $0.35 \pm 0.12^{\mathrm{a}}$ & $3.61 \pm 0.31^{\mathrm{b}}$ & $0.60 \pm 0.06^{\mathrm{a}}$ & $4.11 \pm 1.32^{\mathrm{b}}$ & $0.50 \pm 0.06^{\mathrm{a}}$ & $5.62 \pm 1.97^{\mathrm{b}}$ \\
\hline KIS & $0.54 \pm 0.22^{\mathrm{a}}$ & $2.51 \pm 0.50^{\mathrm{b}}$ & $0.97 \pm 0.30^{\mathrm{a}}$ & $3.44 \pm 1.02^{\mathrm{b}}$ & $0.54 \pm 0.12^{\mathrm{a}}$ & $5.62 \pm 0.77^{\mathrm{b}}$ \\
\hline NAKB & $0.51 \pm 0.16^{\mathrm{a}}$ & $4.02 \pm 1.48^{\mathrm{b}}$ & $1.05 \pm 0.14^{\mathrm{a}}$ & $5.12 \pm 0.63^{b}$ & $0.61 \pm 0.06^{\mathrm{a}}$ & $4.79 \pm 0.30^{b}$ \\
\hline KAZ & $0.68 \pm 0.10^{\mathrm{a}}$ & $3.88 \pm 1.19^{\mathrm{b}}$ & $1.24 \pm 0.15^{\mathrm{a}}$ & $5.39 \pm 1.23^{\mathrm{b}}$ & $0.88 \pm 0.22^{\mathrm{a}}$ & $6.14 \pm 0.63^{b}$ \\
\hline \multicolumn{7}{|c|}{ Juice bananas } \\
\hline KISB & $2.67 \pm 0.49^{\mathrm{a}}$ & $9.36 \pm 1.28^{\mathrm{b}}$ & $2.95 \pm 0.49^{\mathrm{a}}$ & $15.85 \pm 2.23^{b}$ & $2.61 \pm 0.34^{\mathrm{a}}$ & $14.41 \pm 1.24^{b}$ \\
\hline NDI & $1.38 \pm 0.67^{\mathrm{a}}$ & $9.54 \pm 1.33^{\mathrm{b}}$ & $2.67 \pm 0.24^{\mathrm{a}}$ & $18.22 \pm 3.73^{b}$ & $2.24 \pm 0.20^{\mathrm{a}}$ & $20.44 \pm 0.98^{b}$ \\
\hline KAY & $4.90 \pm 0.83^{\mathrm{a}}$ & $12.65 \pm 1.60^{\mathrm{b}}$ & $5.29 \pm 0.56^{\mathrm{a}}$ & $18.33 \pm 1.66^{b}$ & $4.30 \pm 0.56^{\mathrm{a}}$ & $19.15 \pm 1.07^{b}$ \\
\hline
\end{tabular}

Data is compared along rows: Values with different superscripts along the same row are significantly different $(\mathrm{P}<0.05)$. S.P $=$ Spoiled sample.

\section{Discussion of Results}

\subsection{Textural Hardness of the Raw Bananas}

Hardness of bananas in general is a reflection of their eating quality. According to current results, raw juice banana cultivars were significantly harder by about 59\% than cooking banana cultivars (Table 1). In their experiment on "Prediction of textural attributes using color values of banana (Musa sapientum) during ripening", Jaiswal et al. (2014) reported that the initial pulp firmness of banana fruits (at 0 days) was $38.04 \mathrm{~N}$ which decreased to $14.00 \mathrm{~N}$ on the $10^{\text {th }}$ day as the banana ripening. The initial firmness value of $38.04 \mathrm{~N}$ is close to the hardness of juice bananas used in this study. Jaiswal et al. (2014) possibly used desert or juice bananas which fall within this range of firmness. Baoxiu et al. (2000) working on desert banana (green mature Cavendish) obtained hardness of about $22 \mathrm{~N}$ for the raw bananas which is close to the values obtained for cooking bananas in our study. Cavendish is a non-cooking banana whose hardness is expected to be higher than that of regular cooking bananas. Probably the bananas used by Baoxiu et al. (2000) were obtained from commercial sources when they were beginning to ripen, a process that would have reduced their firmness. In our previous work, the same juice bananas used in this study were found to have higher starch, crude pectin and dry matter content but lower moisture than cooking bananas (unpublished results) which may explain why juice bananas are harder as observed in this study. NDI also called "apple banana" is both a juice and a desert banana cultivar and was found to be harder than all banana cultivars investigated in this study. In our day to day experiences, green or unripe juice and desert bananas are generally harder than cooking bananas in both raw and cooked form which supports this observation. Textural hardness is influenced by, banana cultivar and variety, maturity stage, growth season, soils, etc. Current results indicate that hardness of all cooking bananas was not significantly different $(\mathrm{P}<0.05)$; however, in practice these banana cultivars differ in their hardness when cooked, which means that small measurement differences may have significant textural implications when cooked. Ugandan consumers tend to prefer banana cultivars such as KIB and NAKY for softer texture and may dislike MUS and others for harder texture especially during the dry season. This study was conducted during the wet season of April and May 2017.

As results indicate, there were noticeable differences and similarities between cultivars in the different clone sets as grouped by Karamura, (1998). This may not be conclusive since banana classification was largely based on growth and physical characteristics of the different banana cultivars. Results of this study showed that hardness of the selected banana cultivars in each clone set was close which may be related to differences in their genetic composition.

\subsection{Effect of Boiling, Steaming and Mashing on the Textural Hardness}

Boiling and steaming caused a rapid decrease in textural hardness in the first $30 \mathrm{~min}$ and a little thereafter. Mashing in combination with steaming led to significant reduction in hardness relative to steaming alone. The rate of softening of plant tissues during heating follows first-order kinetics (Harada \& Paulus, 1987). This is also 
in agreement with observations made by Baoxiu et al. (2000) who studied the effect of cooking on banana and plantain texture. The observed decrease in hardness of bananas during cooking (boiling and steaming) implies absorption of water by banana starch which on heating hydrates and swells. Then the starch granule structure collapses due to melting of the crystallites, unwinding of the double helices, breaking up of hydrogen bonds hence gelatinization of starch. Cooking also causes alteration of organelles in the cytoplasm and swelling of the cell walls (Xu, Yu, \& Li, 2015; Prestamo, Fuster, \& Risueno, 1998) leading to a gradual breakdown of the protoplasmic structure. This breakdown causes subsequent loss of turgor pressure and a softening effect which is related to changes in the pectic polymers of the cell wall and the middle lamella (Xu et al., 2015). According to Waldron, Parker, \& Smith (2003) the high-temperature exposure during industrial thermal processing and/or home cooking of vegetables causes cell separation, which is related to the solubilization of pectic components, often accompanied by the swelling of cell walls. Starch has a significant influence on the textural properties of foods. When heated in water, starch granules become hydrated, swell, and are transformed into a paste. These structural changes are accompanied by loss of the characteristic birefringence of intact starch granules (as reviewed by Wang, Li, Copeland, Niu, \& Wang, 2015).

Boiling produced the softest texture followed by mashing and steaming (Tables 2a, 2b, 2c and Table 3). This is probably because boiling provided bananas with adequate access to water relative to steaming. Boiling bananas in plenty of water probably increases their water content due to osmotic exchange (Dadzie \& Orchard, 1997). Gouado et al. (2011) reported that after boiling, the water content of unripe and ripe bananas increases significantly $(\mathrm{p}<0.05)$. Excess water, at prolonged heating enhances swelling and gelatinization of starch and further enhances breakdown and solubilization of other intercellular materials such as pectin leading to structural separation. Depending on water availability, time of exposure and temperature, higher degrees of swelling and gelatinization of starch and pectin can be achieved, hence lowering textural hardness. Mashing resulted in intermediate hardness while steaming produced the hardest texture in hot cooked form. Mashing causes structural breakdown of the native bonds within the banana structural matrix which exposes starch and pectin to more moisture hence increased uptake of water and gelatinization. Access to water during cooking is in ascending order - boiling $>$ mashed/steaming $>$ steaming. Steaming restricts the amount of moisture in contact with the bananas which in turn limits starch gelatinization. Boiled cooking bananas were observed to disintegrate in water relative to their steamed counterparts, which remained firm and intact during the whole process of cooking. This re-affirms observations made by Ambigaipalan, Hoover, Donner, \& Liu, (2013) showing that the amount of water available influences the degree of starch gelatinization. Current results showed that boiling produced the softest bananas followed by "mashing/steaming" and lastly steaming alone.

Current results also showed that juice bananas were harder than cooking bananas under all the three cooking treatments used i.e. boiling, steaming and "mashing". This means that the tendency for banana starch to gelatinize and cause structural breakdown during cooking is more pronounced in cooking bananas than in juice bananas. It is not well established why juice bananas are generally harder than cooking bananas. This may probably be related to the native water, starch, pectin \& dry matter content and degree of molecular compactness which may restrict the extent and rate of water penetration into the banana structural matrix to cause swelling and tissue separation. In our previous studies, juice bananas used in this study were found to have more starch, pectic substances and dry matter content but lower moisture than cooking bananas (unpublished results). In our day-to-day experience, juice (beer) and desert bananas at green mature stage are much harder relative to cooking bananas both in raw and cooked form which is one reason why juice bananas are not cooked for meals. It is important to recognize that each of the banana varieties both cooking and juice were different in hardness re-affirming what consumers normally experience with the different varieties of bananas during utilization. These differences in firmness loss are due to inherent differences in cell size and structure, as well as differences in chemical composition before cooking (Dadzie, 1998)

\subsection{Effect of Cooking Time on Textural Hardness}

It was noted that bananas cooked for a shorter time were significantly harder than banana cooked for a longer time. This was particularly more pronounced when bananas were allowed to cool. Increased cooking time allows bananas to absorb more water which increases the extent of starch and pectin gelatinization. This probably enhances extensive structural breakdown, enables tissues to gain and retain significantly high moisture levels, and reduces their propensity to lose it during cooling. This in turn keeps banana starch gelatinized longer and hence softer.

\subsection{Effect of Cooling on Textural Hardness}

All cooked bananas significantly hardened on cooling $(\mathrm{P}<0.05)$ (Table 3$)$. The observed textural hardening of 
cooked bananas during cooling could be attributed to retrogradation of starch. Starch in form of disaggregated amylose and amylopectin chains in a gelatinized starch paste normally undergoes re-association to form more ordered crystalline structures (Wang et al., 2015). On cooling, the disaggregated starch chains retrograde gradually into partially ordered structures that differ from those in native starch granules which may have detrimental effects on the quality of starch-rich foods like bananas. According to Wang \& Copeland (2013), the quality and nutritional properties of starch-based foods are largely determined by the changes that starch undergoes during processing and subsequent storage. These changes involve water uptake, granule swelling, formation of a viscoelastic paste during heating, followed by re-association of dispersed starch chains on cooling and formation of a firm gel which probably is responsible for textural hardening in cooked bananas. The extent of hardening may be influenced by the degree of retrogradation which is in turn influenced by storage temperature, water content of the gelatinized starchy food, starch source, extent of gelatinization of the starch, and amylopectin chain length (Ambigaipalan et al., 2013; Fu, Wang, Li, Zhou, \& Adhikari, 2013). For instance, the low water content may have limited the degree of starch gelatinization in juice bananas hence the hard texture. This is further observed in banana samples boiled or steamed for a shorter time of $30 \mathrm{~min}$, which were harder than those cooked for a longer time of $130 \mathrm{~min}$.

Upon cooling, mashed bananas were harder than steamed and boiled bananas. It is possible that mashing and pressing of the bananas led to creation of new bonds upon cooling that are stronger than the native bonds. Pressing may cause bonds to become shorter and stronger which also excludes air spaces in the banana structural matrix making the product more compact and harder on cooling.

Results showed that a greater part of textural hardening occurred in the first hour of cooling, a trend that was observed in all banana varieties. This implies that hardening of bananas during cooling follows "first order kinetics". Wang et al. (2015) reported retrogradation of starch to be rapid initially which slowed down thereafter.

\section{Conclusions and Recommendations}

This study has shown that cooking reduced hardness. Juice banana cultivars were harder than cooking banana cultivars under similar treatments irrespective of cooking time. Among juice bananas, Kayinja was the hardest in cooked form while in raw form, Ndiizi was the hardest. Among cooking bananas, Kibuzi was the softest while Nakabululu, Kazirakwe, Kisansa and Musakala were among the hardest both in raw and cooked forms. Steaming resulted in a harder texture of cooked bananas relative to mashing and boiling. Therefore, this study recommends boiling of bananas for best texture. If bananas are steamed, the process should be coupled with mashing for a softer texture. Cooked bananas rapidly hardened in the first hour upon cooling and mashed bananas were the hardest hence should be consumed in less than $30 \mathrm{~min}$ of serving when texture is still soft. Bananas cooked longer had lower hardness regardless of cooking method. Therefore, bananas should be cooked for at least $70 \mathrm{~min}$ to guarantee soft texture particularly with steaming and mashing.

Factors responsible for differences in textural hardness between juice and cooking bananas need further investigation. Molecular structural changes that occur in bananas when cooked and cooled under different cooking treatments need probing to clarify the key changes undergone by macromolecules comprising bananas.

\section{Acknowledgements}

This work was funded by the African Development Bank (AfDB) under the AfDB - V- HEST Project under the Ministry of Education and Sports. Authors are very grateful to Prof. Kaaya A., Prof. Byaruhanga Y.B., and Mr. Balamaze J. of Food Science and Nutrition Department, Makerere University for their technical support.

\section{References}

Almazan, A. (1990). Influence of plantain and cooking banana cultivar and ripeness on processed product quality. Journal of Food quality, 13, 351-359. https://doi.org/10.1111/j1745-4557.1990.tb00031.x

Ambigaipalan, P., Hoover, R., Donner, E., \& Liu, Q. (2013). Retrogradation characteristics of. Food research International, 54, 203-12. https://doi.org/10.1016/j.foodres.2013.06.012

Amin, M., Hossain, M., Rahim, M., \& Uddin, M. (2015). Determination of optimum maturity stage of banana. Bangaladesh Journal of Agricultural Research, 40(2), 189-204. https://doi.org/10.3329/bjar.v40i2.24557

Bagaud, C., Chillet, M., Beaute, M., \& Dubois, C. (2006). Physicochemical analysis of mountain bananas from French West Indies. 108(2), 167-172.

Bakare, A., Ogunbowale, O., Adegunwa, M., \& Olusanya, J. (2016). Effects of pretreatments of banana (Musa AAA,Omini) on the composition, rheological properties, and baking quality of its flour and composite blends with wheat flour. Food Science and Nutrition, 5(2), 182-196. https://doi.org/10.1002/fsn3.378 
Baoxiu, Q., Moore, K., \& Orchard, J. (2000). Effect of cooking on banana and plantain texture. Journal of Agricultural Food Chemistry, 48(9), 4221-4226. https://doi.org/10.1021/jf991301z

Chen, L., \& Opara, U. (2013a). Approaches to analysis and modeling texture in fresh and processed foods - A review. Journal of Food Engineering, 119(3), 497-507. https://doi.org/10.1016/j.jfoodeng.2013.06.028

Dadzie, B. (1998). Post-harvest characteristics of black Sigatoka resistant banana, cooking banana and plantain hybrids. Technical guidelines 4. Rome, Italy: International Plant Genetic Resources Institute .

Dadzie, B. K., \& Orchard, J. E. (1997). Routine post-harvest screening of banana/plantain hybrids: criteria and methods. Rome, Italy: International Plant Genetic Resources Institute (IPGRI).

Dury, S., Bricas, N., Tchango-Tchango, J., \& Bikoi, A. (2002). The Determinants of Urban Plantain consumption in Cameroon. Journal of Food Quality and Preference, 13(2), 81-88. https://doi.org/10.1016/S0950-3293(01)00061-1.

Dzomeku, B., Bam, R., Adu-kwarteng, E., Darkey, S., \& Ankomah, A. (2007a). Agronomic and physio-chemical evaluation of FhIA-21 in Ghana. International Journal Agric. Research, 2, 92-96.

Edmeades, S., Smale, M., \& Karamura, D. (2006). Biodiversity of Bananas on Farms in Uganda. Genetic Resource Policies Promising Crop Biotechnologies for Smallholder Farmers in East Africa: Bananas and Maize. International Food Policy Research Institute, 24.

Food and Agricultral Organization of the United Nations (FAO). (2018). Banana facts and figures. Retrieved from http://www.fao.org/economic/est/est-commodities/bananas/bananafacts/en/\#.W0XePToza1s.

Fu, Z., Wang, L., Li, D., Zhou, Y., \& Adhikari, B. (2013). The effect of partial gelatinization of corn satrch on its retrogradation. Carbohydrate Polymer, 97, 512-7. https://doi.org/10.1016/j.carbopol.2013.04.089

Gouado, I., Demasse, M., Etame, L., Meyimgo, O. R., Ejoh, A., \& Fokou, E. (2011). Impact of three cooking methods (Steaming, Roasting on charcoal and Frying) on the beta-carotene and vitamin C contents of plantain and sweet potato. American Journal of Food Technology, 6(11), 994-1001. https://doi.org/10.3923/ajft.2011.994.1001

Harada, T., \& Paulus, K. (1987). Effects of cooking treatments on texture of root vegetables. Agric. Biol. Chem., 51(3), 837-844. https://doi.org/10.1080/00021369.1987.10868110

Jaiswal, P., Jha, S., Kaur, P., Bhardwaj, R., Singh, A., \& Wadhawan, V. (2014). Prediction of textural attributes using color values of banana (Musa sapientum) during ripening. Journal of Food Science and Technology, 51(6), 1179-1184. https://doi.org/10.1007/s13197-012-0614-2

Jha, S., Sethi, S., Srivastav, M., Dubey, A., Sharma, R., Samuel, D., et al. (2010a, March). Firmness characteristics of mango hybrids under ambient storage. Journal of Food Engineering, 97(2), 208-212. https://doi.org/10.1016/j.jfoodeng.2009.10.011

Kabahenda, M., \& Kapiriri, M. (2010). Analysing the Agricultural Science and Technology Innovation Systems: A Case Study of the Banana Sub-Sector in Uganda. Resume. The Technical Centre for Agricultural and Rural Cooperation (CTA).; RUFORUM. Kampala, Uganda: Agricultural Science and Technology Innovation Systems.

Karamura, D. (1998). Numerical taxonomic studies of the East African Highland bananas (Musa AAA-East Africa) in Uganda. Montpellier, France.

Kaur, S., Singh, N., Sodhi, N., \& Rana, J. (2009). Diversity in properties of seed and flour of kidney bean germplasm. Food Chemisry, 117(2), 282-289.

Nalunga, A., Kikulwe, E., Nowakunda, K., Ajambo, S., \& Naziri, D. (2015). Structure of the Cooking Banana Value Chain in Uganda and Opportunities for Value Addition and Postharvest Losses Reduction. National Agricultural Research Laboratories, Kampala, Uganda; Bioversity International, Kampala, Uganda; International Potato Center (CIP), Kampala, Uganda. Kamapala, Uganda: CGIAR. Research Program on Roots, Tubers and Bananas.

Paciulli, M., Ganino, T., Pellegrini, N., Rinaldi, M., Zaupa, M., ... Fabbri, A. (2016, May). Effect of different cooking methods on structure and quality of industrially frozen carrots. Journal of food science and technology, 53(5), 2443-51. https://doi.org/10.1007/s13197-016-2229-5

Perrier, X., De langhe, E., Donohue, M., Lentfer, C., Vrydaghs, L., ... Bakry, F. (2011). Multidisciplinary perspectives on banana (Musa spp.) domestication. Proc. Natl. Acad. Sci. U.S.A., 108, 11311-11318. 
https://doi.org/10.1073/pnas.1102001108

Prestamo, G., Fuster, C., \& Risueno, M. (1998). Effect of blanching and freezing on the structure of carrot cells and their implications for food processing. Journal of the Science of Food and Agriculture, 77(2), 223-229. doi.org/10.1002/(SICI)1097-0010(199806)77:2<223::AID-JSFA29>3.0.CO;2-2

Price, N. (1999). Highland bananas in Colombia (Vol. 8).

Setiady, D., Tang, J., Younce, F., Swanson, B., \& Clary, C. (2009). Porosity, color, texture and microscopic structure of russet potatoes dried using microwave vacuum, heated air and freeze drying. American Society of Agricultural and Biological Engineers, 25(5), 719-724.

The Portal for Statistics (Statista). (2018). Production volume of bananas worldwide from 2010 to 2016. Retrieved from https://www.statista.com/statistics/264001/worldwide-production-of-fruit-by-variety

Waldron, K., Parker, M., \& Smith, A. (2006). Plant cell walls and food quality. . Comprehensive reviews in Food Science and Food Safety, 2(4), 128-146. https://doi.org/10.1111/j.1541-4337.2003.tb00019.x

Wang, S., \& Copeland, L. (2013). Molecular disassembly of starch granules during gelatinization and its effect on starch digestibility: a review. Food Function, 4, 1564-80. https://10.1039/c3fo60258c.

Wang, S., Li, C., Copeland, L., Niu, Q., \& Wang, S. (2015). Starch Retrogradation: A comprehensive Review. Institute of Food Technologists, 14(5), 568-585. https://doi.org/10.1111/1541-4337.12143

Xu, C., Yu, C., \& Li, Y. (2015). Effect of blanching pretreatment on carrot texture attribute, rheological behavior and cell structure during cooking process. LWT-Food Science and Technology, 62(1), 48-54. https://doi.org/10.1016/j.lwt.2015.01.033

Yang, Z., Zheng, Y., Cao, S., Tang, S., Ma, S., \& Li, N. (2007, February). Effects of storage temperature on textural properties of Chinese bayberry fruit. Journal of Textural Studies, 38(1), 166-177.

https://doi.org/10.1111/j.1745-4603.2007.00092.x

\section{Copyrights}

Copyright for this article is retained by the author(s), with first publication rights granted to the journal.

This is an open-access article distributed under the terms and conditions of the Creative Commons Attribution license (http://creativecommons.org/licenses/by/4.0/). 